\title{
Discovery of a strong Baldwin effect in mid-infrared AGN lines ${ }^{\star}$
}

\author{
S. F. Hönig ${ }^{1}$, A. Smette ${ }^{2}$, T. Beckert ${ }^{1}$, H. Horst ${ }^{3,4}$, W. Duschl ${ }^{3}$, P. Gandhi ${ }^{5}$, M. Kishimoto ${ }^{1}$, and G. Weigelt ${ }^{1}$ \\ 1 Max-Planck-Institut für Radioastronomie, Auf dem Hügel 69, 53121 Bonn, Germany \\ e-mail: shoenig@mpifr-bonn.mpg.de \\ 2 European Southern Observatory, Casilla 19001, Santiago 19, Chile \\ 3 Institut für Theoretische Physik und Astrophysik, Christian-Albrechts-Universität zu Kiel, Leibnizstr. 15, 24098 Kiel, Germany \\ 4 Zentrum für Astronomie, ITA, Universität Heidelberg, Albert-Ueberle-Str. 2, 69120 Heidelberg, Germany \\ RIKEN Cosmic Radiation Lab, 2-1 Hirosawa, Wakoshi Saitama 351-0198, Japan \\ Received 5 May 2008 / Accepted 14 May 2008
}

\section{ABSTRACT}

\begin{abstract}
We present the discovery of a Baldwin effect in 8 nearby Seyfert galaxies for the three most prominent mid-infrared forbidden emission lines observable from the ground that are commonly found in AGN, [Ar III] $(\lambda 8.99 \mu \mathrm{m})$, [S IV] $(\lambda 10.51 \mu \mathrm{m})$, and [Ne II] $(\lambda 12.81 \mu \mathrm{m})$. The observations were carried out using the VLT/VISIR imager and spectrograph at the ESO/Paranal observatory. The bulk of the observed line emission originates in the innermost region within a diameter of 0.4 , which corresponds to spatial scales of less than $100 \mathrm{pc}$ within the targeted galaxies. The correlation index is approximately -0.6 and does not vary significantly for all lines studied. To date, this is the strongest anticorrelation that has been measured between line equivalent width and continuum luminosity. In the case of Circinus, we show that, despite using mid-infrared lines, obscuration by either the host galaxy or the circumnuclear dust torus might affect the measurement. Given the small observed spatial scales from which most of the line emission emanates, it is unclear how well these observations agree with the favored "disappearing NLR" scenario for the narrow-line Baldwin effect.
\end{abstract}

Key words. galaxies: Seyfert - galaxies: active - infrared: galaxies - galaxies quasars: emission lines - X-rays: galaxies

\section{Introduction}

The Baldwin effect, a negative correlation between equivalent width, $W_{\lambda}$, and continuum luminosity, $L$, is commonly found in broad UV/optical emission lines of AGN. Although initially reported for the CIV( $\lambda 1549)$ line (Baldwin 1977) for which $W_{\lambda} \propto L^{-0.2}$, it was later found also in the emission lines of many other species for which the strength of the correlation depended on the ionization potential of the respective line (e.g. Dietrich et al. 2002). Although the Baldwin effect is well-established, its physical origin is still a matter of debate. A common explanation refers to the spectral shape of the ionizing continuum, which appears softer in high-luminosity sources than in AGN of lower luminosities. This produces, relative to the AGN luminosity, a lower number of ionizing photons in the broad-line region (BLR), which generates, in turn, a smaller $W_{\lambda}$. While this explanation agrees, at least qualitatively, with the observed dependence on line ionization energy, it is unclear whether $L$ is the fundamental parameter driving the broad-line Baldwin effect (BLBE). A similar effect was observed when $L$ was replaced with the black hole mass, $M_{\mathrm{BH}}$, or the Eddington ratio, $l_{\text {Edd }}=L / L_{\text {Edd }}$ (e.g. Wandel 1999; Warner et al. 2004).

In addition to the BLBE, several narrow optical emission lines were found to display a Baldwin effect with similar order-of-magnitude luminosity-dependence (hereafter: oNLBE; e.g. Brorsen \& Green 1992; McIntosh et al. 1999; Croom et al. 2002). Given the different spatial scales corresponding to broad- and narrow-line emission, it is commonly assumed that the physical mechanisms responsible for the BLBE and oNLBE are different (e.g. Shields 2007, and references therein).

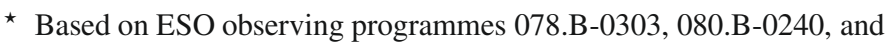
the DDT program 280.B-5068.
}

A luminosity-dependence of the equivalent width, sometimes referred to as the Iwasawa-Taniguchi effect, is known also for the narrow K $\alpha$-line in X-rays (e.g. Iwasawa \& Taniguchi 1993; Bianchi et al. 2007).

We present our discovery of a strong Baldwin effect in nearby Seyfert galaxies for the most prominent narrow emission lines present in AGN mid-infrared spectra and observable from the ground, in particular [Ar III] $(\lambda 8.99 \mu \mathrm{m}),[\mathrm{S} \mathrm{IV}](\lambda 10.51 \mu \mathrm{m})$, and $[\mathrm{Ne} I \mathrm{II}](\lambda 12.81 \mu \mathrm{m})$. The Baldwin effect in these midinfrared forbidden lines (hereafter: iNLBE) is far more pronounced than for any other as yet observed. In Sect. 2, we describe our VLT/VISIR observations. In Sect. 3, our results are presented, followed by a discussion of these findings in Sect. 4. This paper presents first results on line emission of our larger campaign using VLT/VISIR, which is dedicated to midinfrared spectroscopy of nearby AGN at high spatial resolution. A forthcoming paper (hereafter: Paper II) will deal with the midinfrared continuum emission of AGN. In the following, we use the cosmological parameters $H_{0}=73 \mathrm{~km} \mathrm{~s}^{-1} \mathrm{Mpc}^{-1}, \Omega_{\Lambda}=0.72$, and $\Omega_{\mathrm{m}}=0.24$ (Spergel et al. 2007).

\section{Observations}

We used the mid-infrared imager and spectrograph VISIR mounted on the $8 \mathrm{~m} \mathrm{UT3}$ telescope of the ESO/Paranal observatory. With VISIR, we observed 8 nearby AGN of which 5 are Seyfert 2 (S1h, S1i, S2) and 3 are Seyfert 1 (S1.0, S1.5) galaxies. The $8-13 \mu \mathrm{m} N$-band spectra were acquired in low spectral-resolution mode $(R \sim 300)$, for which 4 different wavelength settings centered at $8.5,9.8,11.4$, and $12.4 \mu \mathrm{m}$ have to be combined for each object. With VISIR, the achieved spatial resolution in this wavelength range is $0.25-0.39$, which is a 


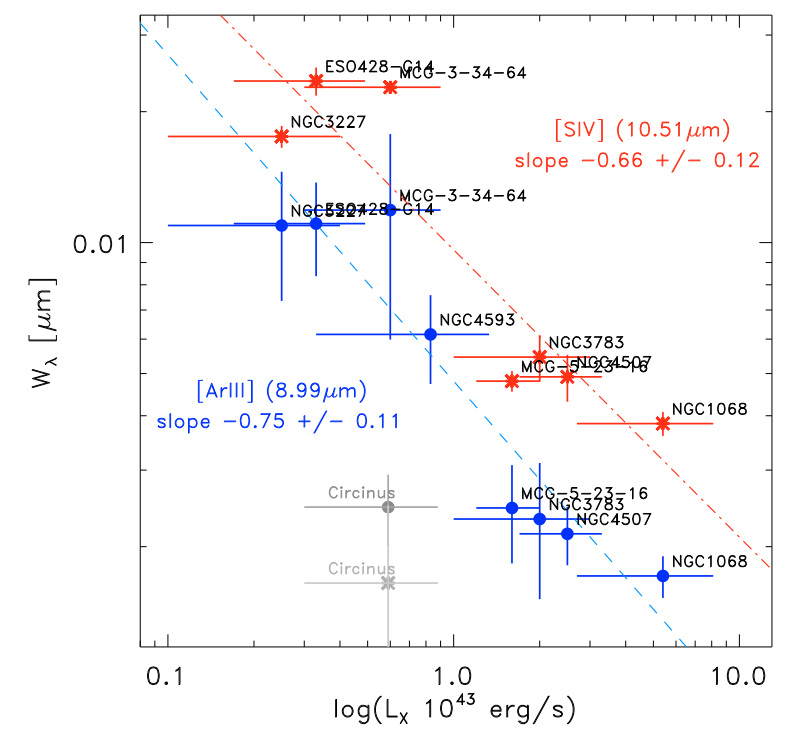

Fig. 1. Equivalent width, $W_{\lambda}$, of the [Ar III] (blue circles) and [S IV] (red crosses) lines plotted over the X-ray luminosity, $L_{\mathrm{X}}$, for our sample of nearby AGN as listed in Table 1 . The fitted correlations $W_{\lambda} \propto L_{\mathrm{X}}^{-0.75 \pm 0.11}$ for [Ar III] (blue dashed line; $\rho_{\text {Spearman }}=-0.91$, significance 0.002), and $W_{\lambda} \propto L_{\mathrm{X}}^{-0.66 \pm 0.12}$ for [S IV] (red dashed-dotted line; $\rho_{\text {Spearman }}=-0.79$, significance 0.04 ) are shown. Circinus (gray symbols) is an outlier probably because of significant dust obscuration in the host galaxy or the circumnuclear dust torus (see Fig. 4).

factor 2-4 smaller than the used slit widths of $0.75-11^{\prime \prime} 0$, making slit losses negligible. The spectra were extracted using the standard VISIR CPL pipeline (V3.0.0) and calibrated using a number of generic standard stars. For NGC 4507, a single standard star observed during a single night was used for calibration. In addition to the 8 objects, archival VISIR data of NGC 1068 and NGC 253 has been downloaded, extracted, and calibrated in the same way. A more detailed description of the observations will be presented in Paper II. NGC 4593 was observed only in the 8.5 and $9.8 \mu \mathrm{m}$ settings, corresponding to a restframe wavelength coverage of approximately $7.9-10.4 \mu \mathrm{m}$. As a result, only $[\mathrm{Ar}$ III] $(\lambda 8.99 \mu \mathrm{m})$ is seen in the combined spectrum, providing $W_{\lambda}$ data of this line for a total of 8 objects. For both $[\mathrm{S}$ IV $](\lambda 10.51 \mu \mathrm{m})$ and $[\mathrm{Ne} \mathrm{II}](\lambda 12.81 \mu \mathrm{m})$, the equivalent widths of 7 objects are available.

\section{The Baldwin effect in forbidden mid-IR lines}

\subsection{Results}

In Table 1 , we present extracted $W_{\lambda}$ measurements for all three lines. For all objects, the line emission was strongly peaked towards the nucleus. Apart from emission in the most nearby objects NGC $1068(\sim 10 \%$ extension in [S IV] with respect to the continuum PSF) and Circinus ( $\sim 50 \%$ extension in [S IV] and $[\mathrm{Ne} \mathrm{II}]$ ), no spatial extension was detected in the continuum and the lines. The data reduction was completed so that even for these extended objects, almost no line flux is lost by the extraction window. The errors in $W_{\lambda}$ represent the uncertainty due to the shape determination of continuum emission, underneath the emission line. This uncertainty was, in part, due to the presence of prominent sky lines in those spectra, which were taken under unfavorable atmospheric conditions (e.g. large amount of precipitable water vapor). The measurements of [Ne II] line emission was affected the most because strong atmospheric bands were present between $12.4-12.7 \mu \mathrm{m}$. On the other hand, the

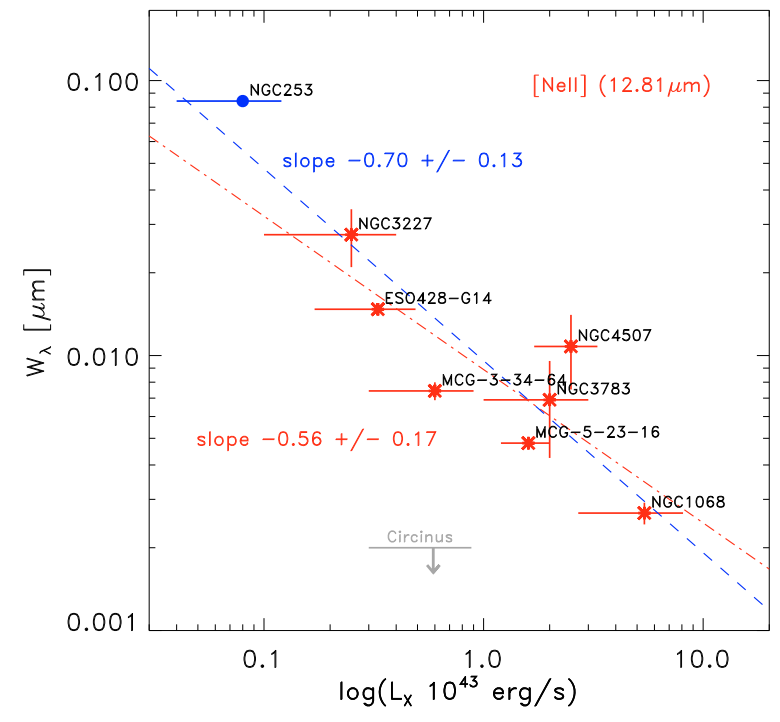

Fig. 2. Equivalent width, $W_{\lambda}$, of the [Ne II] line plotted versus the X-ray luminosity, $L_{X}$ for 7 nearby Seyfert galaxies (red crosses). The fitted correlation $W_{\lambda} \propto L_{\mathrm{X}}^{-0.56 \pm 0.17}\left(\rho_{\text {Spearman }}=-0.75\right.$, significance 0.05$)$, is shown as a red, dash-dotted line. When including NGC 253 (blue circle) - despite its questionable AGN nature - a correlation $W_{\lambda} \propto L_{\mathrm{X}}^{-0.70 \pm 0.13}$ ( $\rho_{\text {Spearman }}=-0.83$, significance 0.01 ) is found (blue dashed line). An upper limit to the measurement for Circinus (gray arrow) is given. The non-detection of [Ne II] from the nuclear point source is probably due to significant dust obscuration in either the host galaxy or circumnuclear dust torus (see Fig. 4).

error bars of the [Ar III] line are caused predominantly by the line weakness with respect to its continuum.

In addition to the line $W_{\lambda}$ 's, Table 1 lists the (absorptioncorrected) $2-10 \mathrm{keV}$ X-ray luminosities, $L_{\mathrm{X}}$, for our sample of AGN. These are interpreted as a measure of the ionizing continuum luminosity, since the UV/optical AGN emission of our type 2 sources is heavily affected by dust absorption from the putative circumnuclear dust torus. Unfortunately, 3 of our objects are Compton-thick to the X-ray emission (NGC 1068, Circinus, and ESO 428-G14). For these objects, we estimated the nuclear X-ray luminosity by extracting the $12 \mu \mathrm{m}$ continuum emission from our spectra (which will be presented in Paper II), and translating the corresponding spectral luminosity into $L_{X}$ using the $L_{\mathrm{MIR}}-L_{\mathrm{X}}$-correlation for AGN established by VISIR observations of similar spatial resolution (Horst et al. 2008). We adopt an error of $50 \%$ in $L_{X}$ for these objects.

In Figs. 1 and 2, we show the measured $W_{\lambda}$ of [Ar III], [S IV], and $\left[\mathrm{Ne}\right.$ II] versus $L_{\mathrm{X}}$. The trend of smaller $W_{\lambda}$ with luminosity is evident. As an illustration, the spectral regions around the [Ar III] and [S IV] lines are presented in Fig. 3 for NGC 3227 $\left(\log L_{X}=42.4\right)$, MCG-5-23-16 $\left(\log L_{X}=43.2\right)$, and NGC 1068 $\left(\log L_{X}=43.7\right)$. A statistical analysis of the correlation for our sample shows Spearman ranks $\rho_{\text {Spearman }}=-0.75 \ldots-0.9$ with significance levels in the range of $0.2-5 \times 10^{-2}$. Despite the limited number of objects and luminosity coverage, the correlations that are clearly evident in data can be confidently established. For BLBE and oNLBE studies, higher significance is achieved by averaging spectra of different objects within a narrow luminosity range. This accounts for peculiarities in individual objects (e.g. Croom et al. 2002). We aim to acquire observations for a larger sample to complete similar studies of the presented iNLBE. We note that no correlation of $W_{\lambda}$ with AGN distance, $D_{\mathrm{L}}$, is present in our sample $\left(\rho_{\text {Spearman }}<0.25\right.$, significance $>0.6$ for all lines). 
Table 1. Equivalent widths and X-ray luminosities of our sample of nearby AGN.

\begin{tabular}{|c|c|c|c|c|c|c|c|c|}
\hline object & type $^{a}$ & $\begin{array}{c}D_{\mathrm{L}}^{b} \\
(\mathrm{Mpc})\end{array}$ & $\begin{array}{c}10 \mu \mathrm{m} \mathrm{scale}^{c} \\
\text { (pc) }\end{array}$ & $\begin{array}{c}W_{\lambda}([\mathrm{Ar} \text { III }]) \\
\left(10^{-4} \mu \mathrm{m}\right)\end{array}$ & $\begin{array}{l}W_{\lambda}([\mathrm{S} \text { IV }]) \\
\left(10^{-4} \mu \mathrm{m}\right)\end{array}$ & $\begin{array}{c}W_{\lambda}([\mathrm{Ne} \mathrm{II}]) \\
\left(10^{-4} \mu \mathrm{m}\right)\end{array}$ & $\begin{array}{c}L_{\mathrm{X}} \\
\left(10^{43} \mathrm{erg} / \mathrm{s}\right)\end{array}$ & Ref. \\
\hline NGC 1068 & S1h & $14.4^{d}$ & 21.4 & $17.13 \pm 0.94$ & $38.37 \pm 1.21$ & $26.74 \pm 1.21$ & $5.4 \pm 2.7$ & 1 \\
\hline ESO 428-G14 & $\mathrm{S} 2$ & 22.3 & 33.2 & $110.57 \pm 13.43$ & $235.16 \pm 8.74$ & $147.40 \pm 2.44$ & $0.33 \pm 0.16$ & 1 \\
\hline MCG-5-23-16 & S1i & 39.5 & 58.8 & $24.55 \pm 3.12$ & $48.04 \pm 1.30$ & $111.61 \pm 1.46$ & $1.7 \pm 1.0$ & 2 \\
\hline NGC 4507 & S1h & 54.5 & 80.9 & $21.41 \pm 1.64$ & $49.14 \pm 3.03$ & $107.91 \pm 16.32$ & $2.5 \pm 0.8$ & 3 \\
\hline Circinus & S1h & $4.2^{e}$ & 6.2 & $24.66 \pm 2.31$ & $16.50 \pm 4.20$ & $<27$ & $0.59 \pm 0.29$ & 1 \\
\hline MCG-3-34-64 & S1h & 63.3 & 94.2 & $118.78 \pm 29.46$ & $227.63 \pm 1.28$ & $74.35 \pm 2.77$ & $0.6 \pm 0.3$ & 3 \\
\hline NGC 3783 & $\mathrm{~S} 1.5$ & 44.7 & 66.5 & $23.15 \pm 4.00$ & $54.56 \pm 3.33$ & $68.99 \pm 13.28$ & $2.0 \pm 1.0$ & 3 \\
\hline NGC 3227 & $\mathrm{~S} 1.5$ & 20.4 & 30.4 & $109.48 \pm 18.02$ & $175.37 \pm 5.09$ & $275.10 \pm 32.69$ & $0.25 \pm 0.15$ & 4 \\
\hline NGC 4593 & S1.0 & 42.0 & 62.5 & $61.52 \pm 7.19$ & & & $0.83 \pm 0.50$ & 5 \\
\hline NGC 253 & $\mathrm{~S}$ & $3.5^{f}$ & 5.2 & $\ldots$ & ... & $842.27 \pm 11.13$ & $0.08 \pm 0.04$ & 1 \\
\hline
\end{tabular}

Notes: ${ }^{a}$ AGN types from Veron-Cetty \& Veron (2006); ${ }^{b}$ luminosity distance based on CMB reference frame redshifts from NED, otherwise indicated; ${ }^{c}$ diffraction limit for a $8.2 \mathrm{~m}$ VLT telescope $\sim 0.25 ;{ }^{d}$ Tully (1988); ${ }^{e}$ Freeman et al. (1977); ${ }^{f}$ Rekola et al. (2005).

References: (1) $L_{\mathrm{MIR}}-L_{\mathrm{X}}$-correlation from Horst et al. (2008) using $12 \mu \mathrm{m}$ VISIR fluxes for NGC 1068 (14 Jy), Circinus (10 Jy), ESO $428-\mathrm{G} 14$ (0.22 Jy), and NGC 253 (2.1 Jy)); (2) Balestra et al. (2004); (3) Horst et al. (2008); (4) mean of recent 2-10 keV (Cappi et al. 2006) and 17-60 keV (Sazanov et al. 2007) data taken, 60\% error inferred due to variability; (5) Shinozaki et al. (2006).

To date, only one other study has considered a possible Baldwin effect in the mid-infrared. In an AAS abstract, Keremedjiev \& Hao (2006) presented the detection of a Baldwin effect for the [S IV] line in AGN data obtained by the Spitzer satellite. They noted indications of a Baldwin effect for [Ne II], although they conceded that the accuracy of their study was limited by the low spatial resolution of the Spitzer data that they used. In the available abstract, they do not state either the slope or the scatter of the anti-correlation. We demonstrate here, that the Baldwin effect for both the [S IV] and [Ne II] line, and, in addition, the [Ar III] line, is quite significant - and strong - when data of high spatial resolution are used, even for a small number of objects. High spatial resolution data therefore appears to be crucial for the accurate detection of the Baldwin effect in the mid-IR.

\subsection{Relation to $M_{B H}$ and $l_{\text {edd }}$}

For the three type $1 \mathrm{AGN}$ in our sample, black-hole masses, $M_{\mathrm{BH}}$, were estimated using reverberation mapping data. We adopted black-hole masses for NGC $3227\left(\log M_{\mathrm{BH}}\left(M_{\odot}\right)=\right.$ $7.63 \pm 0.31)$ and NGC $3783\left(\log M_{\mathrm{BH}}=7.47 \pm 0.08\right)$ from Onken et al. (2004), and $\log M_{\mathrm{BH}}=6.99 \pm 0.10$ for NGC 4593 from Denney et al. (2006). In addition, the black-hole mass of the Seyfert 2 galaxy NGC 1068 was determined from MASER cloud kinematics data to be $\log M_{\mathrm{BH}} \approx 7.0$ (Greenhill et al. 1996). For these four AGN, we analyzed the dependence of the [Ar III]-line equivalent width on $M_{\mathrm{BH}}$. A statistical test showed no evident correlation for our small number of objects and the narrow range of black hole masses $\left(\rho_{\text {Spearman }}=0.40\right.$, significance 0.6 , for a nominal fit $\left.W_{\lambda}([\mathrm{Ar} \mathrm{III}]) \propto M_{\mathrm{BH}}^{0.5 \pm 0.7}\right)$. Subject to these limitations, this indicates a fundamental difference between the iNLBE and BLBE, for which an anticorrelation was found (Warner et al. 2004).

By applying bolometric corrections to the X-ray luminosities listed in Table 1 (Marconi et al. 2004), it is possible to estimate the Eddington ratio, $l_{\text {Edd }}$, of four AGN with known $M_{\mathrm{BH}}$. A statistical test for a correlation between $W_{\lambda}([\mathrm{Ar}$ III] $])$ and $l_{\text {Edd }}$ reveals a Spearman rank $\rho_{\text {Spearman }}=-0.8$ with a significance of 0.2 for the relation $W_{\lambda}([\mathrm{Ar} \mathrm{III}]) \propto l_{\mathrm{Edd}}^{-0.40 \pm 0.17}$. Our limited data-set size, therefore, does not allow a correlation to be firmly established, but we do measure a negative dependence of $W_{\lambda}$ on $l_{\text {Edd }}$.

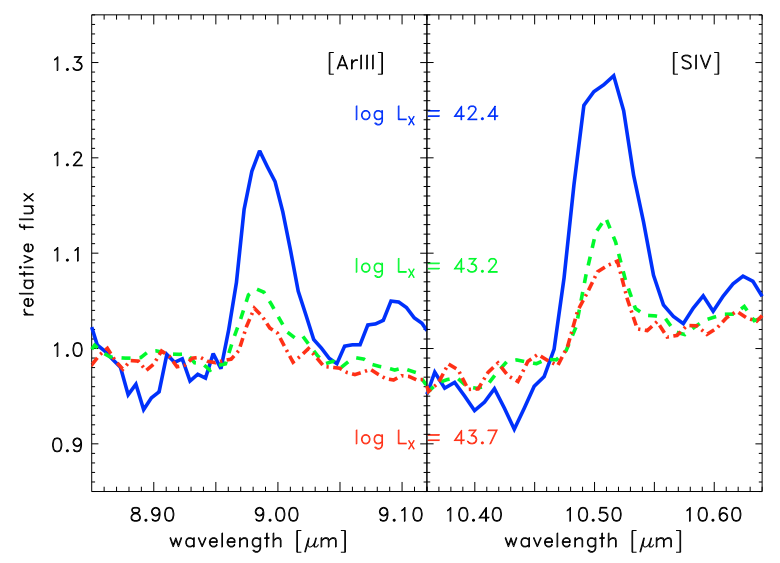

Fig. 3. [Ar III] (left) and [S IV] (right) spectral region of NGC 3227 ( $\log L_{\mathrm{X}}=42.4$; solid blue line), MCG-5-23-16 $\left(\log L_{\mathrm{X}}=43.2\right.$; dashed green line), and NGC $1068\left(\log L_{X}=43.7\right.$; dash-dotted red line) illustrating the decrease of $W_{\lambda}$ with $\mathrm{X}$-ray luminosity. The spectra have been smoothed to flatten the continuum.

\subsection{The case of Circinus}

We excluded Circinus from all of the correlation analyses. As can be seen in Figs. 1 and 2, the $W_{\lambda}$ 's of all three mid-infrared lines for Ciricnus deviate significantly from the nominal fit to the other AGN. Although the discrepancy between its properties and those of the remaining galaxies could be due to a resolution effect (Circinus is the closest AGN in our sample), the data for NGC 1068 does have a similar spatial resolution when the difference in the AGN luminosities is taken into account (using the scaling $r \propto L^{1 / 2}$ ). Moreover, we did not detect extended emission in the lines down to $\sim 5 \%$ of the peak flux. This provides confidence to reject the idea that a significant amount of line flux was over-resolved and lost in the noise.

Circinus is known for strong dust extinction towards the nucleus by dust lanes in both the host and our own Galaxy. In Fig. 4, we plot the deviation from the nominal correlation with X-ray luminosity (as given in Figs. 1 and 2) of each line against the depth of the silicate feature. Circinus shows the strongest deviations from all correlations and, at the same time, has the deepest silicate feature. It is deeper than in any other type 2 AGN in our sample. In the case of foreground extinction (host or our 


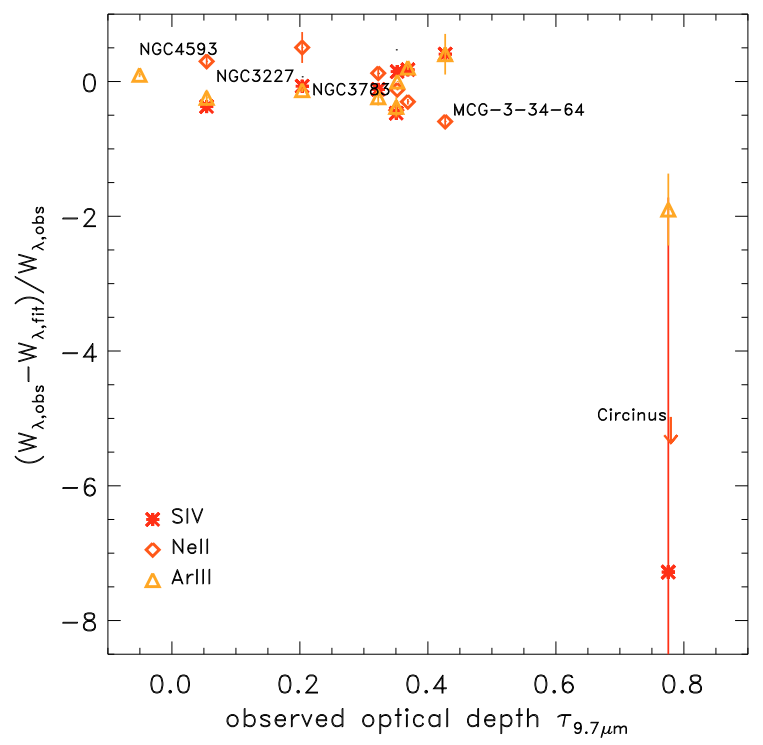

Fig. 4. Relative deviation of the observed $W_{\lambda}$ from the correlation for all three lines plotted against the observed optical depth, $\tau_{9.7 \mu \mathrm{m}}$, in the silicate feature. Circinus, which is an outlier in the Baldwin correlations of all three lines, is the object with the deepest silicate feature. Due to radiative transfer effects, the actual obscuration of the central AGN by dust from the putative torus might be even stronger than suggested by the $\tau_{9.7 \mu \mathrm{m}}$ value.

galaxy), we expect that the flux within the lines is reduced in the same way without a change in $W_{\lambda}$. On the other hand, if part or all of the line emission originates inside of the torus, the extinction in the lines could be far stronger in a more or less edge-on torus geometry. Given the fact that the bulk of line emission in all other AGN originates in an unresolved point source, such a scale and inclination effect appears possible. Strong extinction towards the NLR based on mid-infrared spectra of Circinus was also noted by Roche et al. (2006).

\section{Discussion and conclusions}

\subsection{A comparison to UV/optical lines}

The negative correlations in the [Ar III], [S IV], and [Ne II] lines, observed with VLT/VISIR, is far stronger than any known Baldwin effect in optical/UV broad or narrow lines. This is remarkable since the spatial resolution of optical observations should be similar or even better than that achieved in the midinfrared with the VLT. In UV/optical lines, a trend for larger correlation slopes with increasing ionization potential is observed (e.g. Dietrich et al. 2002). The potential of the presented lines is in the range of $40-47 \mathrm{eV}$ and, thus, comparable to those of the optical/UV lines. From the optical/UV lines, slopes of approximately -0.1 to -0.2 would be expected. The measured slope of the mid-infrared lines deviates by a margin of at least 4- $\sigma$ from expectation (apart from [NeII] without NGC 253, for which the deviation is instead $2-\sigma$ ).

In Sect. 3.2, we found some negative trend of $W_{\lambda}$ ([Ar III] $)$ with $l_{\text {Edd }}$, but possibly not with $M_{\mathrm{BH}}$. If confirmed, this observed trend would be in contrast to the observed and theoreticallyexpected behavior of the broad lines (e.g. Wandel 1999; Warner et al. 2004). Thus, a possible non-existence of a $W_{\lambda}-M_{\mathrm{BH}^{-}}$ correlation might imply that the physical origin of the iNLBE differs from that of the BLBE; this would question, in particular, how relevant the $M_{\mathrm{BH}}$-dependence of the ionizing continuum is for the mid-infrared narrow-line emission.

\subsection{Implications for the origin of the iNLBE}

The physical origin of the oNLBE is possibly related to the size of the narrow-line region (NLR), for which a luminosity dependence $R_{\mathrm{NLR}} \propto L^{0.5}$ was found (Bennert et al. 2002). As a consequence, the NLR of luminous sources may reach galactic-size scales and, therefore, lose its gas enitrely. This scenario is often referred to as the "disappearing NLR" (Croom et al. 2002; Netzer et al. 2006). For the mid-infrared lines, we find that the main portion of their emission does not originate in a spatiallyextended region, as for instance [O III] in the optical, but from spatial scales smaller than $100 \mathrm{pc}$. If the absence of detected line emission in Circinus is an inclination-dependent obscuration effect, then the spatial scales involved in mid-infrared line emission are very small. Since it is difficult to imagine how the NLR could disappear on these scales, the iNLBE probably requires a different explanation. This should explain, in particular, (1) the steepness of the Baldwin effect in the presented lines; and (2) why the mid-infrared lines do not follow the same ionization potential-dependence as observed in optical lines. Possible reasons could involve the presence of a luminosity-dependent increase in density inside the inner NLR, which could surpress forbidden line emission with increasing efficiency at higher luminosity.

Acknowledgements. We thank L. Spinoglio for helpful comments and suggestions which improved the paper. This research made use of the NASA/IPAC Extragalactic Database (NED) operated by the JPL (Caltech), under contract with NASA. P.G. is supported by JSPS and RIKEN Foreign postdoctoral fellowships. HH acknowledges support by DFG via SFB 439.

\section{References}

Baldwin, J. A. 1977, ApJ, 214, 679

Balestra, I., Bianchi, S., \& Matt, G. 2004, A\&A, 415, 437

Bennert, N., Falcke, H., Schulz, H., Wilson, A., \& Wills, B. J. 2002, ApJ, 574, L105

Bianchi, S., Guainazzi, M., Matt, G., \& Fonseca Bonilla, N. 2007, A\&A, 467, L19

Brorsen, T. A., \& Green, R. F. 1992, ApJS, 80, 109

Cappi, M., Panessa, F., Bassani, L., et al. 2006, A\&A, 446, 459

Croom, S. M., Rhook, K., Corbett, E. A., et al. 2002, MNRAS, 337, 275

Denney, K. D., Bentz, M. C., Peterson, B. M., et al. 2006, ApJ, 653, 152

Dietrich, M., Hamann, F., Shields, J. C., et al. 2002, ApJ, 581, 912

Freeman, K. C., Karlson, B., Lynga, J. F., et al. 1977, A\&A, 55, 445

Greenhill, L. J., Gwinn, C. R., Antonucci, R., \& Barvainis, R. 1996, ApJ, 472, L21

Horst, H., Gandhi, P., Smette, A., \& Duschl, W. J. 2008, A\&A, 479, 389

Iwasawa, K., \& Taniguchi, Y. 1993, ApJ, 413, L15

Keremedjiev, M., \& Hao, L. 2006, A\&AS, 20914912

Marconi, A., Risaliti, G., Gilli, R., et al. 2004, MNRAS, 351, 169

McIntosh, D. H., Rieke, M. J., Rix, H.-W., Foltz, C. B., \& Weymann, R. J. 1999, ApJ, 514, 40

Netzer, H., Mainieri, V., Rosati, P., \& Trakhtenbrot, B. 2006, A\&A, 453, 525

Onken, C. A., Ferrarese, L., Merritt, D., et al. 2004, ApJ, 615, 645

Rekola, R., Riecher, M. G., McCall, M. L., et al. 2005, MNRAS, 361, 330

Roche, P. F., Packham, C., Telesco, C. M., et al. 2006, MNRAS, 367, 1689

Sazonov, S., Revnivtsev, M., Krivonos, R., Churazov, E., \& Sunyaev, R. 2007, A\&A, 462, 57

Shields, J. A. 2007, ASPC, 373, 355

Shinozaki, K., Miyaji, T., Ishisaki, Y., Ueda, Y., \& Ogasaka, Y. 2006, AJ, 131, 2843

Spergel, D. N., Bean, R., Doré, O., et al. 2007, ApJS, 170, 377

Tully, R. B. 1988, Nearby Galaxies Catalogue (New York: Cambridge Univ. Press)

Veron-Cetty, M. P., \& Veron, P. 2006, A\&A, 455, 773

Wandel, A. 1999, ApJ, 527, 649

Warner, C., Hamann, F., \& Dietrich, M. 2004, ApJ, 608, 136 